antigen for complement fixation, and the fixation result is negative, and we get a positive cure or material improvement from the elimination of this tooth, I think we are justified in concluding that the primary focus was where we believed, and the complement fixation showed there was no demonstrable toxin in the blood due to this organism.

Now as to Dr. Schamberg's statement about a recurring case of iritis in which he used a vaccine. If he made a careful roentgenographic examination and eliminated the dental focus, where did he get his vaccine for this case? Did he use a stock vaccine?

Dr. Morris I. Schamberg, Nrw York: I refer to the fact that a roentgenographic examination proved negative until I used common sense in conjunction with it and decided to remove a pulpless tooth from which we took a culture.

Dr. Joseph M. Levy, New York: As to animal inoculations, most of the men doing animal experimentation seldom use less than 3 to 5 c.c. of a twenty-four hour culture. Their bacteria will probably run up to 100 million to the cubic centimeter. Rosenow uses colossal quantities, 15 to 30 c.c. We do not get anything like this in the human being. What we get, or what we have been led to believe we get, is a slow; gradual but constant inoculation with the active virus from the original focus. What we are trying to produce in our animals is a similar condition. If we give massive inoculations our rabbits promptly go to postmortem. We are trying to produce these slow but constant injections, simulating the conditions we find in the human subject, and see what results vill be obtained. We have had the usual results obtained by other experimentors as to cardiac, arthritic and other involvements, but to date we have been unable to produce an experimental infectious eye condition.

\section{THE CRUCIAL TEST OF THERAPEUTIC EVIDENCE *}

\section{TORALD SOLLMANN, M.D.} CLEVELAND

According to the good old truism, the last and crucial proof of the pudding is in the eating thereof; and so, the last and crucial test of a therapeutic agent is its consumption by a patient. There is, however, one essential difference: When the pudding is eaten, with a sense of satisfaction, we know that it was a good, or at least an eatable pudding.

If the patient improves after taking a remedy, we do not yet know that he improved on account of the remedy. The post hoc type of reasoning or logic is not respectable; bit it is all too apt to creep in unawares, unless one takes great precautions indeed.

Clinical evidence needs especially to be on its guard against this pitfall, for the conditions of disease never remain constant; nor is it possible to foresee with certainty the direction which they are going to take. It is just this point which makes the clinical evidence so much more difficult to interpret than laboratory evidence, in which the conditions can be more or less exactly controlled, and any changes foreseen. It is on this account, also, that clinical experiments must be surrounded with extra painstaking precautions.

In brief, while the "proof" of a remedy is on the patient, that is not the whole story, but merely an introduction. The real problem is to establish the causative connection between the remedy and the events. The imperfect realization of this has blocked therapeutic advance, has disgusted critical men to the point of therapeutic nihilism, and has fertilized the ground for the commercial exploitation of drugs that are of doubtful value or worse.

\footnotetext{
* Read before the Section on Pharmacology and Therapeutics at the Sixty-Eighth Annual Session of the American Medical Association, New York, Iune, 1917 .
}

This has been impressed on me particularly by my service on the Council on Pharmacy and Chemistry. In the course of its work of passing on the claims advanced for commercial remedies, this council is forced to inquire critically into the basis of the claims of manufacturers.

It is interesting to note the qualitative differences in the evidence for the various kinds of claims: The chemical data are usually presented in such a form that it is possible to tell at a glance whether or not they are based on demonstrated facts, which could usually be.verified or refuted without special difficulty. The deductions are usually such as can be legitimately drawn from the data, or else they are obviously absurd. All this agrees with the relatively exact status of chemical science.

In passing to data and deductions from animal experiments, a distinct change is noticeable: Not only are the data less reliable, and less worthy of confidence, but they are more often stated in a less straightforward manner. The presentation of the data often shows evidence of manipulations of the results, so as to make them most favorable to a preconceived conclusion that would recommend the drug. This is not always intentional, but is partly due to the less exact nature of animal experimentation, which leaves a wider play to the arbitrary interpretation of the reporter. A certain amount of this is unavoidable. No serious objection can be raised, provided the experimenter presents all the essential data, and discusses fairly all of the interpretations that would apply to them.

On the whole, it is usually possible to form a fairly definite estimate of the value of experimental data.

When one comes to the clinical evidence, an entirely different atmosphere obtains. When the Council demands evidence of the usefulness of a remedy, the manufacturers generally respond with every sign of enthusiasm. They may have ready a series of articles already published, or they instruct their agents to bring in letters from physicians. The last method seems to meet the most cordial response, judging from the deluge of letters and opinions that floods the Council.

The quality of the published papers is a fair reflection of the deficiencies of what is still the common type of clinical evidence. A little thought suffices to show that the greater part cannot be taken as serious evidence at all. Some of the data are merely impressions - usually the latest impressions of an impressionable enthusiast - the type of man who does not consider it necessary to present evidence for his own opinions; the type of man who does not even realize that scientific conclusions must be based on objective phenomena.

Some of the papers masquerade as "clinical reports," sometimes with a splendid disregard for all details that could enable one to judge of their value and bearing, sometimes with the most tedious presentation of all sorts of routine observations that have no relation to the problem.

The majority of reports obtained by the agents belong to these classes, notwithstanding the fact that they are often written for the special use of the Council, and therefore with the realization that they are likely to be subjected to a thorough examination, and therefore presumably representing the best type of work of which the reporter is capable. So, at least, one would suppose. 
It is also possible, however, that some of these reports are written merely out of thoughtlessness, or perhaps often to get rid of an importunate agent. This is illustrated by the following correspondence, taken literally from the files of the Council.

A letter from a prominent physician "A," endorsing a certain preparation "D," having been submitted to the Council, the secretary was directed to write to Dr. $A$ as follows:

Dear Dr. A:-The B Company of $\mathrm{C}$ has requested the Council on Pharmacy and Chemistry to admit its preparation $\mathrm{D}$ to New and Nonofficial Remedies. As part evidence for the value of the preparation, the company submitted a letter from you which contains the following:

So far as my experience has thus far gone, they are certainly superior to a number of other iodine compounds now on the market, and I should judge that they ought to take a superior place in therapy involving the use of iodine.

The referee of the Council in charge of $D$ writes that he was interested by your letter and asks that I inquire: As compared with sodium or potassium iodid, what would you say are the differences between, and real advantages of, $D$ and the alkaline iodids? Did you make any comparative experiments and keep a record of them? If so, the referee would like to receive an account of your trials. In what direction could $\mathrm{D}$ be expected to occupy a superior place in iodin therapy?

I hope that you can give the information asked by the referee and thus aid the Council in arriving at a correct estimate regarding the value of $D$.

The following reply was received from the physician in response to the foregoing:

Dear Professor Puckner:-In reply to yours of January 19 I did not proceed far enough in the investigation of $\mathrm{D}$ to draw conclusions of any particular value for the purpose of the Council on Pharmacy and Chemistry; and I so stated in my letter to the proprietors of that remedy.

Answers to the questions you put in your letter require an amount of investigation of the remedy far beyond anything I undertook. As a matter of fact, I returned about five sixths of the capsules sent me, because of lack of time and opportunity to carry out the extensive clinical experiments that I plainly saw would be required to give an opinion at all worth while. I believe you had better not consider me in the matter at all.

The report was furnished by a physician for whom I have a high personal regard. I introduce it here, not so much in a spirit of criticism, but as a justification of the opinion that I have formed of clinical evidence obtained by manufacturers through their clinical adjutors.

When commercial firms claim to base their conclusions on clinical reports, the profession has a right to cxpect that these reports should be submitted to competent and independent review. When such reports are kept secret, it is impossible for any one to decide what proportion of them are trustworthy, and what proportion thoughtless, incompetent or accommodating. However, if this were done it is quite possible that such firms would find much more difficulty in obtaining the reports. Those who collaborate should realize frankly that under present conditions they are collaborating, not so much in determining the scientific value, but rather in establishing the commercial value of the article.

Often the best type of clinical reports - those in which the observations are directed to the significant events and not to mere side lines, and in which the significant events are correctly and adequately reported - generally lack one important essential, namely, an adequate control of the natural course of the disease.
Since this cannot be controlled directly, it must be compensated indirectly, For this purpose, there are available two methods:

The first is the statistical method in which alternate patients receive or do not receive the treatment. This method can usually only be of value when a very large series of patients is available. Even then, its value is limited or doubtful, because it cannot take sufficient account of the individuality of cases.

The second method consists in the attempt to distinguish unknown preparations by their effects - the method that might be called the "comparative method" or the "blind test."

In this, the patient, or a series of patients, is given the preparation which is to be tested, and another preparation which is inactive, and the observer aims to distinguish the two preparations from their effects on the patient. Surely if the drug has any actions at all, it will be possible to select correctly in a decided majority of the administrations.

The same principle can be applied in distinguishing the superiority of one preparation over another. In this case, the two preparations would be given alternately to different patients, and the observer would try to distinguish them by their effects. Here again, if one drug is really superior or otherwise different from another, to a practically important extent, the observer will surely be able to make the distinction.

This method is really the only one that avoids the pitfalls of clinical observation; it is the only method that makes the results purely objective, really independent of the bias of the observer and the patient. It is the only method, therefore, which determines whether it was really the pudding that was eaten and not some other dessert.

In principle this method does not usually offer any very great difficulties. It is, of course, necessary that the two preparations to be compared shall resemble each other so closely or shall be flavored, etc., so that they cannot be distinguished by their physical properties. This is usually not a very difficult matter. The method does not jeopardize the interests of the patient, for it is understood that no drug would be tested in this way unless there is some reason to believe that it has a value. When the patient's condition is such as to demand treatment, then he would be receiving either the standard drug or the drug which the experimenter believes may be superior to the standard.

\section{CONCLUSIONS}

The final and crucial test of a remedy is on the patient; but the test must be framed so as to make it really crucial. Most clinical therapeutic evidence falls far short of this. The "blind test" is urged to meet the deficiencies.

\section{New and Nonofficial Remedies}

THE FOLLOWING ADDITIONAL ARTICLE HAS BEEN ACCEPTED as CONFORMing to the rules of the Council on Pharmacy and Chemistry of the American Medical Association for admission to New and NoNOFficial Remedies. A copy of THE RULES ON WHICH THE COUNCIL BASES ITS ACTION WILl BE sent on application. $\quad$ W. A. Puckner, Secretary.

ACETYLSALICYLIC ACID (See N. N. R., 1917, p. 265), Acetylsalicylic Acid, M. C. W.-A non-proprietary brand complying with the standards for acetylsalicylic acid.

Mallinckrodt Chemical Works, St. Louis, Mo. 